J. Lake Sci. (湖泊科学), 2019, 31(6): 1601-1611

DOI 10. 18307/2019. 0619

(c) 2019 by Journal of Lake Sciences

\title{
三峡库区丰都一忠县段消落带不同高程土壤镉及其形态的分布特征”
}

\author{
钟志淋 $^{1,2}$, 䣓海健 ${ }^{1}$, 吴艳宏 ${ }^{1 * *}$, 祝 贺 ${ }^{1,2}$, 王晓晓 ${ }^{3}$, 周 俊 $^{1}$ \\ (1: 中国科学院、水利部成都山地灾害与环境研究所, 山地表生过程与生态调控重点实验室,成都 610041) \\ (2:中国科学院大学,北京 100049) \\ (3:西华师范大学国土资源学院,南充 637000)
}

\begin{abstract}
摘 要: 镉 $(\mathrm{Cd})$ 是目前三峡库区消落带主要的污染物之一, 其迁移和形态转化直接威胁水环境安全. 选择库区丰都一忠 县段消落带,按照高程差异分别于不同土地利用类型(农田、林地、果园和村庄)下采集土壤样品,利用化学连续提取法测 定 $\mathrm{Cd}$ 的形态组成, 探讨影响消落带土壤中 $\mathrm{Cd}$ 及其形态空间分布的关键因素. 结果表明, 消落带土壤中 $\mathrm{Cd}$ 的平均含量为 $0.65 \mathrm{mg} / \mathrm{kg}$, 可交换态及碳酸盐结合态是土壤中 $\mathrm{Cd}$ 的主要形态, 占比达到 $41.21 \%$, 残渣态和铁锰氧化物结合态次之, 有机 物及硫化物结合态占比最低. 不同土地利用类型下消落带土壤 $\mathrm{Cd}$ 的含量整体上不存在显著性差异, 表明当地人类活动 对土壤 $\mathrm{Cd}$ 的直接贡献较弱. 消落带土壤 $\mathrm{Cd}$ 及其形态的含量与高程呈显著负相关, 且在 $160 \sim 165 \mathrm{~m}$ 区域发生明显转变, 水位变化导致的泥沙沉积可能是控制消落带 $\mathrm{Cd}$ 空间分布的主要因素. 此外, 土壤理化性质, 光其是细颗粒泥沙对 $\mathrm{Cd}$ 及 其形态分布具有明显影响,将来需重点关注泥沙理化属性对 $\mathrm{Cd}$ 迁移及形态转化的影响.
\end{abstract}

关键词: 重金属;形态组成;土地利用类型;水位变化;三峡水库;消落带

\section{Distribution of cadmium in soils along the altitude of riparian zone (Fengdu-Zhongxian section) in the Three Gorges Reservoir region}

\author{
ZHONG Zhilin ${ }^{1,2}$, BING Haijian ${ }^{1}$, WU Yanhong ${ }^{1 * *}$, ZHU He ${ }^{1,2}$, WANG Xiaoxiao ${ }^{3} \&$ ZHOU Jun $^{1}$ \\ (1: Key Laboratory of Mountain Surface Processes and Ecological Regulation, Institute of Mountain Hazards and \\ Environment, Chinese Academy of Sciences, Chengdu 610041, P.R.China) \\ (2: University of Chinese Academy of Sciences, Beijing 100049, P.R.China) \\ (3: China West Normal University, Land and Resources School, Nanchong 637000, P.R.China)
}

\begin{abstract}
Cadmium( Cd) is one of the major pollutants in the water-level fluctuation zone of the Three Gorges Reservoir region. The migration and fraction transformation of Cd directly threat the safety of water environment. At the riparian zones from Fengdu to Zhongxian in the Three Gorges Reservoir, soil samples were collected according to altitudinal difference under four different land use types including farmland, woodland, orchard and residential zone. The fraction composition of Cd in the soils was determined by chemical sequential extraction. The key factors affecting the spatial distribution of $\mathrm{Cd}$ and its fractions in the riparian soils were deciphered. The results showed that the average content of $\mathrm{Cd}$ in the soil was $0.65 \mathrm{mg} / \mathrm{kg}$. The acid extractable fraction $(41.21 \%$ ) of Cd was the dominant fraction in the soils, followed by residual, reducible and the oxidizable fractions. In general, there is no significant difference in the concentrations of $\mathrm{Cd}$ and its fractions in the soils under different land use types, indicating slight local anthropogenic contribution to $\mathrm{Cd}$ in the riparian soils. Both the content of $\mathrm{Cd}$ and its fractions in the soils showed a significant negative correlation with altitude, and their content had a marked change at the altitude of 160-165 m. This indicates that sediment deposition caused by water-level fluctuation may be the main factor controlling the spatial distribution of Cd in the riparian soils. The physicochemical properties of soils, especially the fine particles, induce an important influence on the spatial distribution of Cd in the riparian soils. Further research should be focused on the effects of sediment properties on the migration and fraction transfor-
\end{abstract}

* 中国科学院青年创新促进会项目 (2017424) 资助. 2019-03-12 收稿;2019-05-28 收修改稿. 钟志淋 (1994 ), 女, 硕士研究生;E-mail: zz1766@ imde.ac.cn.

** 通信作者; E-mail: yhwu@imde.ac.cn. 
mation of $\mathrm{Cd}$ in the riparian soils.

Keywords: Heavy metal; fraction composition; land use type; water-level variation; Three Gorges Reservoir; riparian zones

镉 $(\mathrm{Cd})$ 是一种非人体必需, 且具有较大生物毒性的重金属元素, 能通过食物链产生生物富集和放大效 应, 具有较大的潜在生态环境风险. 同时, $\mathrm{Cd}$ 也是水环境中最危险的污染物质之一, 近年来我国发生的几起 重大 Cd 污染事件均造成了较大的环境危害和经济损失 ${ }^{[1-3]}$. 水环境中 $\mathrm{Cd}$ 的来源包括自然来源与人为来源, 自然来源主要是流域内岩石风化与土壤侵蚀, 人为来源包括工业 “三废”、农药化肥和生活废弃物的排放. 进 人水体中的 $\mathrm{Cd}$ 经过溶解、吸附/解吸、配合、络合等反应后, 会形成多种溶解态和颗粒态的化合物. $\mathrm{Cd}$ 的溶 解态与颗粒态化合物能够被水体中的泥沙所吸附, 当环境条件发生改变时 (如温度升高、 $\mathrm{pH}$ 降低等) 会引起 沉积物 $\mathrm{Cd}$ 的再次释放,容易造成水体的二次污染. 因此, 泥沙既是 $\mathrm{Cd}$ 的“汇”,也是 $\mathrm{Cd}$ 的源 ${ }^{[4]}$.

沉积环境的变化直接关系到沉积物中 $\mathrm{Cd}$ 的迁移转化及其对水环境质量的影响, 目前已有大量的报道. 例如, 细颗粒物质由于具有更大的比表面积和更高的阳离子交换能力, 通常能吸附更多的 $\mathrm{Cd}^{[5]}$; 有机质是土 壤或沉积物中 $\mathrm{Cd}$ 的重要结合相, 能够通过吸附或络合的形式降低 $\mathrm{Cd}$ 的生物有效性和溶解性 ${ }^{[6-7]}$, 而有机质 降解时则会促进 $\mathrm{Cd}$ 向水体的释放 ${ }^{[8]}$; 氧化还原条件对沉积物中 $\mathrm{Cd}$ 的迁移转化能力具有重要影响, 当土壤 或沉积物处于氧化环境时, 硫化物加速氧化, 有机体降解, 进而促进了 $\mathrm{Cd}$ 的释放 ${ }^{[9]}$, 反之亦然. 此外, $\mathrm{pH}^{[10]}$ 、 酸挥发性硫化物 ${ }^{[1-12]}$ 等也对土壤或沉积物中 $\mathrm{Cd}$ 的迁移转化具有重要影响. 因此, 对于水环境而言, 要弄清 $\mathrm{Cd}$ 的环境行为需要综合理解不同理化性质的影响.

消落带是由于江河、湖泊、水库等水体因水位季节性涨落所形成的干湿交替的水陆交错带 ${ }^{[13]}$, 三峡水库 于 2010 年开始正常运行, 水位在 $145 \sim 175 \mathrm{~m}$ 之间反季节变化, 形成了 $349 \mathrm{~km}^{2}$ 消落带 ${ }^{[14]}$. 库坝建设会显著 改变河流流态, 使泥沙沉积加速 ${ }^{[15]}$. 三峡水库进出库水文观测资料显示 ${ }^{[16]}$, 三峡水库泥沙淤积量约 16.38 亿吨, 其中消落带上的淤积量占总量的 7.0\%. 泥沙是河流沉积物中重金属的主要携带者和缓冲剂. 由于三 峡库区消落带泥沙淤积量大、反季节水位调控下沉积环境变化频繁且剧烈, 自水库开始蓄水以来, 消落带土 壤和沉积物中重金属的环境问题已经受到广泛关注. 例如, 有学者调查了消落带土壤中重金属的赋存特征, 发现随着水库平稳运行, $\mathrm{Cd} 、 \mathrm{Cu} 、 \mathrm{~Pb} 、 \mathrm{Zn}$ 等重金属元素的累积呈现增加趋势 ${ }^{[17-20]}$. 其中, 消落带土壤中 $\mathrm{Cd}$ 的 累积较为突出, 其浓度远高于重庆地区土壤背景值, 而且 $\mathrm{Cd}$ 主要存在于非残渣态中, 具有较高的活性 ${ }^{[21-23]}$. 此外, 有研究发现消落带的物源主要为坡岸近缘侵蚀和雨季泥沙沉降 ${ }^{[24]}$, 说明坡岸处的人类活动方式和库 区水位变化对消落带土壤中 $\mathrm{Cd}$ 的累积可能具有重要影响. 但是, 目前少有研究直接关注不同土地利用类型 和海拔差异共同影响下消落带土壤中 $\mathrm{Cd}$ 及其形态的赋存特征. 综上, $\mathrm{Cd}$ 在三峡库区消落带土壤中的累积 程度和有效性均较高, 具有 “二次释放” 的风险. 因此, 系统研究消落带土壤中 Cd 及其形态的赋存特征及关 键控制因素对库区水环境质量具有重要意义.

本研究选择三峡库区丰都一忠县的典型消落带, 通过分析不同土地利用类型和海拔差异下土壤中 $\mathrm{Cd}$ 的 含量及形态, 以期认识不同高程和不同土地利用类型下消落带土壤中 $\mathrm{Cd}$ 及其形态的赋存特征; 揭示影响土 壤中 $\mathrm{Cd}$ 及其形态分布的主要影响因素, 为三峡库区的水环境安全提供理论支撑.

\section{1 材料与方法}

\section{1 研究区概况与样品采集}

选择三峡库区中部丰都县与忠县的典型消落带作为研究区 (图 1). 三峡库区丰都一忠县段的消落带岸 线长且面积大, 是库区泥沙淤积的重点区域. 该区域属于亚热带湿润季风气候, 年平均气温 $18.5^{\circ} \mathrm{C}$, 年均降 雨量约 $1200 \mathrm{~mm}$. 土壤类型以发育自紫色砂岩的紫色土为主. 2017 年 8 月, 选择土地利用类型不同的 4 个消 落带, 4 种土地利用类型分别为农田 (种植水稻)、果园 (种植柑橘)、林地 (人工侧柏林) 和村庄 (图 1). 在每 种土地利用类型下, 按照 $5 \mathrm{~m}$ 的高程间隔设置样品采样点. 将 $150 \sim 175 \mathrm{~m}$ 高程视作消落带区域, $180 \mathrm{~m}$ 高程 视作未受消落带水位变化影响的背景点. 在每个采样点分别布设 3 个 $2 \mathrm{~m} \times 2 \mathrm{~m}$ 的样方, 于每个样方中采集 表层土壤样品 (深度为 $20 \mathrm{~cm}$ ), 由于林地与村庄处的现场环境复杂, 未采集到 $175 \mathrm{~m}$ 高程的样品. 采集后的 样品放置于密封袋中, 保存运输至实验室待测. 


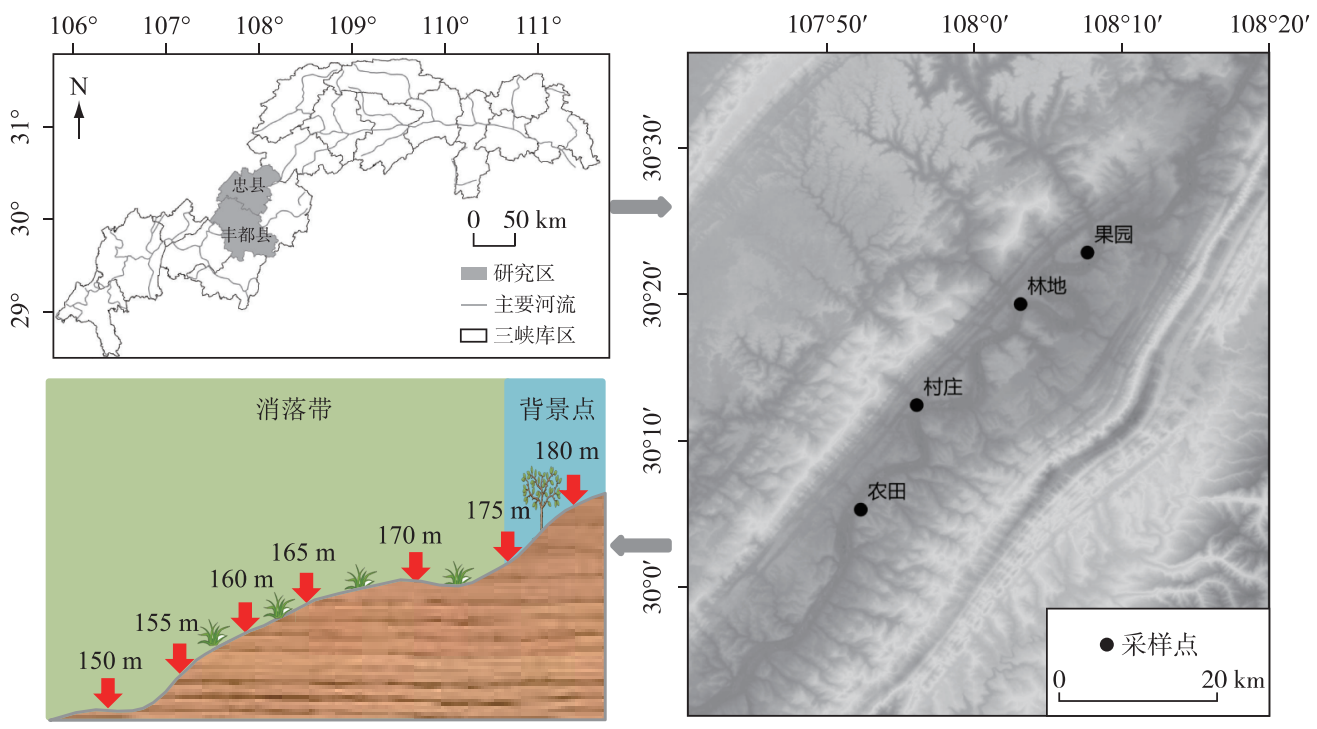

图 1 研究区概况及样点分布

Fig.1 The information of the study area and the distribution of sampling sites

\section{2 样品分析}

土壤 $\mathrm{pH}$ 值采用 $\mathrm{pH}$ 计测定, 水土比为 $2.5: 1$. 有机质含量采用烧失量的方法, 即 $105^{\circ} \mathrm{C}$ 烘干后的样品再 通过马弗炉 $550^{\circ} \mathrm{C}$ 灼烧, 最后计算质量差. 样品粒度采用 Marlven 2000 激光粒度仪测定, 定义直径 $<4 \mu \mathrm{m}$ 颗 粒为黏粒, 直径位于 $4 \sim 64 \mu \mathrm{m}$ 间为粉粒, 直径 $>64 \mu \mathrm{m}$ 为砂粒. 样品采用硝酸一氢氟酸一高氯酸一盐酸法消 解 ${ }^{25]}$, 消解液中 $\mathrm{Cd}$ 的含量利用电感耦合等离子质谱仪 (Inductively Coupled Plasma Mass Spectrometry, ICP$\mathrm{MS}$, 美国) 测定, $\mathrm{Al} 、 \mathrm{Ca} 、 \mathrm{Fe} 、 \mathrm{Mg} 、 \mathrm{Mn} 、 \mathrm{P}$ 和 $\mathrm{Ti}$ 的含量使用电感耦合等离子体原子发射光谱( Inductively Coupled Plasma-Atomic Emission Spectrometer, ICP-AES, 美国)测定. 通过分析平行样品、空白样品和标准物质 (土壤 标准样品为 GBW-07405) 进行质量控制; ICP-MS 和 ICP-AES 分析的测试精度的相对标准偏差 (RSD) 分别在 $5 \%$ 和 3\%以内,标准物质回收率变化范围均在 $95 \% \sim 105 \%$ 之间. 采用 BCR 连续提取法 ${ }^{[26-27]}$, 测定土壤中 $\mathrm{Cd}$ 的可交换态及碳酸盐结合态 (F1)、铁镇氧化物结合态 (F2)、有机物及硫化物结合态 (F3) 和残渣态 (F4) 含 量, 通过分析平行样品和空白样品进行质量控制.

\section{3 数据分析与统计}

采用单因素方差分析法 (One-way ANOVA, Fisher test, $P<0.05)$ 与非参数检验 (Nonparametric tests, Kruskal-Wallis test, $P<0.05)$ 分析各理化性质及 $\mathrm{Cd}$ 在不同组间是否存在差异. $\mathrm{Cd}$ 与其它变量的关联性利用 相关分析法和回归分析法分析, 并采用 $R^{2}$ 和 $t$ 检验分别对回归方程的拟合优度及参数进行检验. 上述分析 通过 SPSS 19.0 及 Origin 8.0 软件完成.

\section{2 结果}

\section{1 消落带土壤的理化性质}

消落带与背景点土壤的理化性质见表 1. 消落带土壤的粒度组成以粉粒为主 (均值 \pm 标准误为 $67.35 \% \pm$ $1.29 \%$,下同), 黏粒 $(16.12 \% \pm 0.49 \%)$ 和砂粒 $(16.53 \% \pm 1.53 \%)$ 的含量相对较低. 土壤的粒度组成在高程间 存在显著性差异 $(P<0.05)$, 随高程上升, 黏粒与粉粒含量减少, 砂粒含量增多. 消落带土壤的粒度组成在 4 种土地利用类型间也存在显著差异 $(P<0.05)$, 农田、果园、林地下方土壤中值粒径为 $15.56 \pm 0.79 \mu \mathrm{m}$, 而村庄 下方的土壤显著粗于其它 3 种土地利用类型 (中值粒径为 $46.25 \pm 10.89 \mu \mathrm{m})(P<0.05)$. 在背景点, 土壤中粉 粒 $(65.29 \% \pm 2.21 \%)$ 含量较高, 砂粒 $(19.48 \% \pm 2.66 \%)$ 次之, 黏粒 $(15.23 \% \pm 1.13 \%)$ 含量最低. 背景点土壤粒 
度组成在 4 种土地利用类型间不存在显著性差异 $(P>0.05)$.

消落带和背景点土壤 $\mathrm{pH}$ 偏中性 (分别为 $7.41 \pm 0.10$ 和 $7.23 \pm 0.27$ ), 并且在不同土地利用类型间与高程 间均不存在显著性差异 $(P>0.05)$. 消落带与背景点土壤有机质含量分别为 $4.23 \% \pm 0.17 \%$ 和 $3.59 \% \pm 0.33 \%$. 消落带土壤有机质含量在不同土地利用类型间无显著性差异 $(P>0.05)$, 但在不同高程间存在显著性差异 $(P<0.05)$, 表现出随高程增加有机质含量升高的趋势. 背景点土壤有机物含量在不同土地利用类型间不存 在显著性差异 $(P>0.05)$.

消落带土壤主要元素 $(\mathrm{Al} 、 \mathrm{Ca} 、 \mathrm{Fe} 、 \mathrm{Mg} 、 \mathrm{Mn} 、 \mathrm{P} 、 \mathrm{Ti})$ 含量在土地利用类型间均无显著性差异 $(P>0.05)$, 但在 不同高程间均存在显著性差异 $(P<0.05)$, 各元素浓度均呈现随海拔升高而下降的趋势 $(P<0.05)$. 背景点土 壤样品 $\mathrm{Al} 、 \mathrm{Mg}$ 和 $\mathrm{Mn}$ 含量在不同土地利用类型间存在显著性差异 $(P<0.05)$, 而其它元素在 4 种土地利用类 型间无显著性差异 $(P>0.05$, 表 1$)$.

\section{2 消落带土壤中 $\mathrm{Cd}$ 及其形态的空间分布特征}

消落带土壤 Cd 含量为 $0.11 \sim 1.34 \mathrm{mg} / \mathrm{kg}(0.65 \pm 0.04 \mathrm{mg} / \mathrm{kg})$, 背景点土壤 $\mathrm{Cd}$ 含量为 $0.13 \sim 1.05 \mathrm{mg} / \mathrm{kg}$ $(0.36 \pm 0.05 \mathrm{mg} / \mathrm{kg}$ ) (图 2, 表 2). 在 4 种土地利用类型下, 消落带和背景点土壤中 $\mathrm{Cd}$ 含量均无显著性差异 $(P>0.05)$. 消落带土壤 $\mathrm{Cd}$ 含量在不同高程间存在显著性差异 $(P<0.05)$, 表现为 150 和 $155 \mathrm{~m}$ 高程土壤 $\mathrm{Cd}$ 含量最高, $160 \mathrm{~m}$ 次之, $165 、 170$ 和 $175 \mathrm{~m}$ 高程最低 $(P<0.05), \mathrm{Cd}$ 的含量与高程间呈现极显著的负相关关系 (表 $3, P<0.01$ ).

消落带土壤中 $\mathrm{Cd}$ 的各形态浓度为 $\mathrm{F} 1(0.30 \pm 0.02 \mathrm{mg} / \mathrm{kg})>\mathrm{F} 4(0.18 \pm 0.01 \mathrm{mg} / \mathrm{kg})>\mathrm{F} 2(0.13 \pm 0.01 \mathrm{mg} / \mathrm{kg})>$ $\mathrm{F} 3(0.03 \pm 0.003 \mathrm{mg} / \mathrm{kg})(P<0.05$, 表 2). 在 4 种土地利用类型间, 土壤 Cd-F1 和 Cd-F2 的含量不存在显著性 差异 $(P>0.05)$, 而 $\mathrm{Cd}-\mathrm{F} 3$ 和 $\mathrm{Cd}-\mathrm{F} 4$ 间存在显著性差异, 其中在村庄处土壤 $\mathrm{Cd}-\mathrm{F} 3$ 的含量显著高于其它土地 利用类型下的水平 $(P<0.05)$, 林地处土壤 Cd-F4 的含量显著高于其它土地利用类型下的水平 $(P<0.05)$. 此 外, 土壤 $\mathrm{Cd}$ 的形态浓度均表现出随海拔上升逐渐降低的趋势 $(P<0.05)$.

消落带土壤中各形态 $\mathrm{Cd}$ 占比为 $\mathrm{F} 1(41.21 \% \pm 1.48 \%)>\mathrm{F} 4(34.71 \% \pm 1.88 \%)>\mathrm{F} 2(19.15 \% \pm 0.77 \%)>\mathrm{F} 3$ $(5.13 \% \pm 0.53 \%)(P<0.05$, 图 2). 在不同土地利用类型间, 各形态 $\mathrm{Cd}$ 的比例均存在显著性差异 $(P<0.05)$, 即, 村庄处 Cd-F1 占比显著高于农田 $(P<0.05)$, 果园与村庄处 Cd-F2 占比显著高于林地 $(P<0.05), \mathrm{Cd}-\mathrm{F} 3$ 占 比为村庄 $>$ 农田 $>$ 果园 $>$ 林地 $(P<0.05)$, 林地与农田处 Cd-F4 占比显著高于村庄 $(P<0.05)$. 在不同海拔间, Cd-F2 与 Cd-F3 占比无显著性差异 $(P>0.05)$, 而 Cd-F1 与 Cd-F4 的占比表现出相反的特征, 高程 $150 、 155$ 和 $160 \mathrm{~m}$ 处 Cd-F1 占比显著高于 $165 、 170$ 和 $175 \mathrm{~m}$ 处 $(P<0.05)$, 而 Cd-F4 占比显著低于 $165 、 170$ 和 $175 \mathrm{~m}$ 处 $(P<0.05)$.

背景点土壤各形态 $\mathrm{Cd}$ 含量表现为 $\mathrm{F} 1(0.14 \pm 0.02 \mathrm{mg} / \mathrm{kg})$ 与 $\mathrm{F} 4(0.13 \pm 0.02 \mathrm{mg} / \mathrm{kg})$ 高于 $\mathrm{F} 2(0.08 \pm 0.01$ $\mathrm{mg} / \mathrm{kg})$ 和 $\mathrm{F} 3(0.02 \pm 0.01 \mathrm{mg} / \mathrm{kg})(P<0.05)$. 村庄处土壤 Cd-F2 和 Cd-F3 含量显著高于其它土地利用类型 $(P<0.05), \mathrm{Cd}-\mathrm{F} 1$ 和 Cd-F4 含量在不同土地利用类型间不存在显著性差异 $(P>0.05)$. 背景点土壤 $\mathrm{Cd}$ 的形态 占比为 $\mathrm{F} 1(39.20 \% \pm 1.46 \%)>\mathrm{F} 4(34.93 \% \pm 1.77 \%)>\mathrm{F} 2(21.33 \% \pm 1.45 \%)>\mathrm{F} 3(4.80 \% \pm 1.29 \%)(P<0.05)$. 果 园和林地处土壤 $\mathrm{Cd}-\mathrm{F} 1$ 占比显著高于村庄 $(P<0.05)$, 村庄处土壤 $\mathrm{Cd}-\mathrm{F} 2$ 占比显著高于林地 $(P<0.05)$, 其它 2 种 $\mathrm{Cd}$ 形态占比在不同土地利用类型间不存在显著性差异 $(P>0.05)$.

\section{3 土壤 $\mathrm{Cd}$ 与理化性质的关系}

Spearman 相关分析的结果表明 (表 3), 消落带土壤中 $\mathrm{Cd}$ 及其形态与黏粒和砂粒呈显著正相关 $(P<$ $0.05)$, 与砂粒呈显著负相关 $(P<0.05)$. 土壤 $\mathrm{Cd}$ 及其形态与 $\mathrm{pH}$ 不存在显著的相关关系 $(P>0.05)$. 土壤总 $\mathrm{Cd} 、 \mathrm{Cd}-\mathrm{F} 1$ 和 Cd-F3 与有机质呈显著负相关 $(P<0.05), \mathrm{Cd}-\mathrm{F} 2$ 和 Cd-F4 与有机质无显著相关性 $(P>0.05)$. 土 壤主要元素 $(\mathrm{Al} 、 \mathrm{Ca} 、 \mathrm{Fe} 、 \mathrm{Mg} 、 \mathrm{Mn} 、 \mathrm{P} 、 \mathrm{Ti})$ 与 $\mathrm{Cd}$ 及其形态呈现极显著的正相关关系 $(P<0.01$, 表 3$)$. 背景点土壤 $\mathrm{Cd}$ 与理化性质的相关分析结果表明 (表 3), $\mathrm{Cd}$ 只与 $\mathrm{Ti}$ 存在显著的相关关系 $(P<0.05), \mathrm{Cd}-\mathrm{F} 1$ 与 $\mathrm{Fe} 、 \mathrm{P}$ 和 Ti 呈极显著正相关 $(P<0.01), \mathrm{Cd}-\mathrm{F} 2$ 与 $\mathrm{Fe}$ 和 $\mathrm{Ti}$ 呈显著相关 $(P<0.05), \mathrm{Cd}-\mathrm{F} 3$ 和 $\mathrm{Cd}-\mathrm{F} 4$ 与各理化指标的关系 均不显著 $(P>0.05)$.

由于 $\mathrm{Cd}$ 的分布在高程间存在显著性差异, 根据 $\mathrm{Cd}$ 及其形态在消落带的海拔分布特征, 定义 $150 \sim 160$ $\mathrm{m}$ 为消落带下部, 165 175 $\mathrm{m}$ 为消落带上部, 分别分析消落带上部和下部土壤 $\mathrm{Cd}$ 及其形态与理化性质的关 


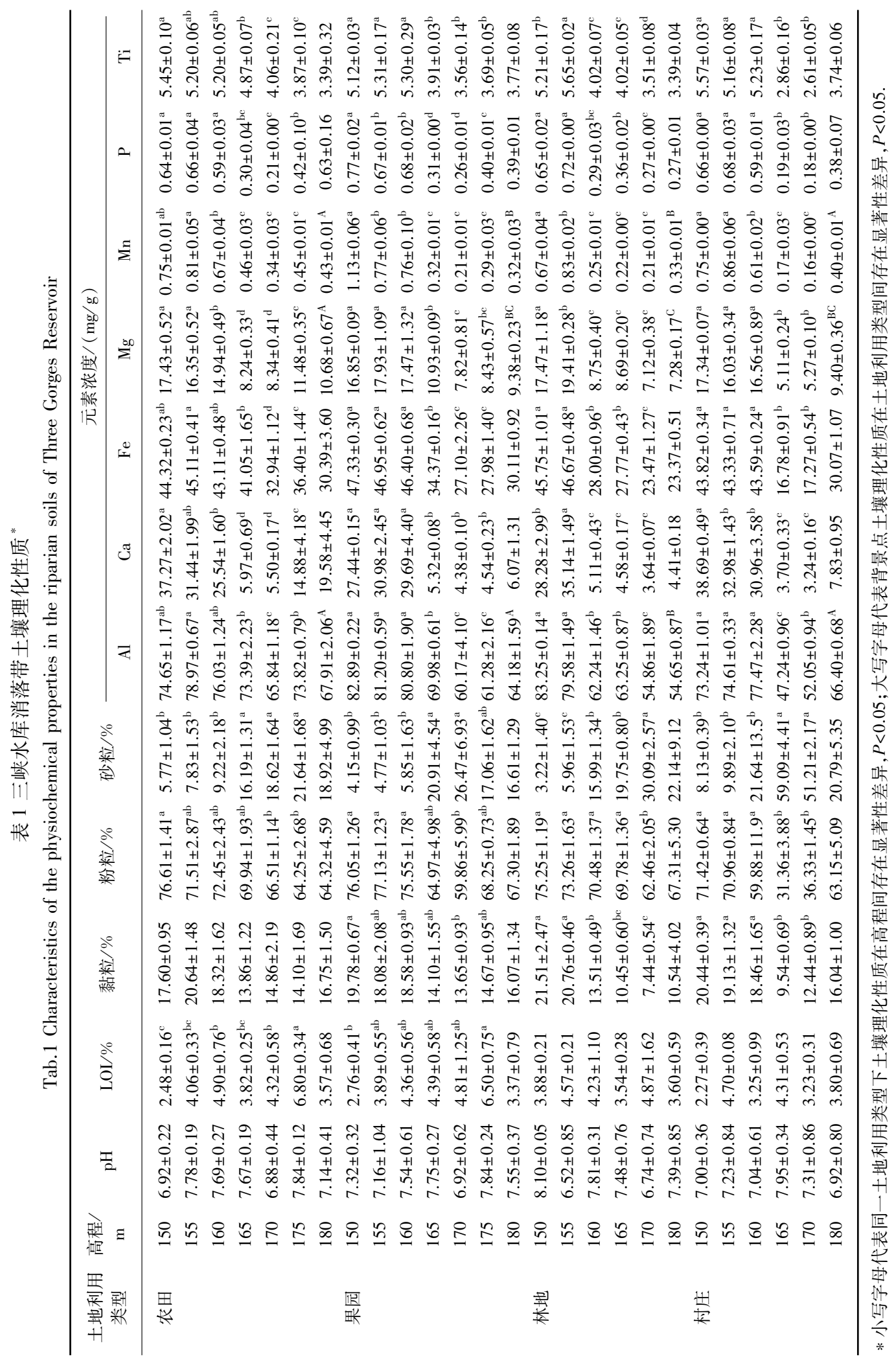



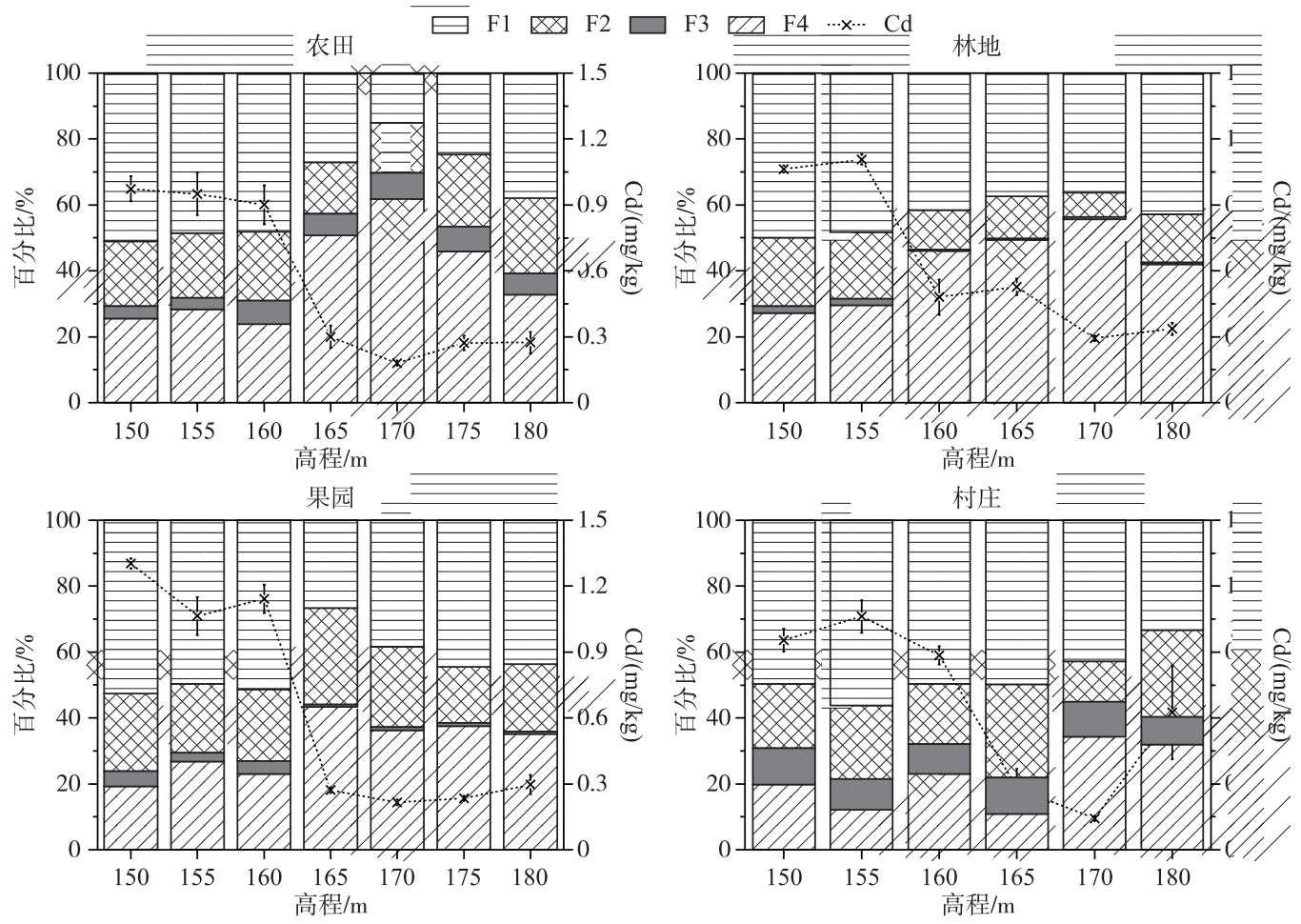

图 2 消落带土壤 $\mathrm{Cd}$ 及其形态含量的空间分布

Fig. 2 The spatial distribution of Cd content and its fractions in the riparian soils

系 (表 3). 与消落带上部相比, 消落带下部土壤理化性质与 $\mathrm{Cd}$ 及其形态的关系更加密切, 土壤砂粒、砂粒、 主要元素 (除 Ti 以外) 与 $\mathrm{Cd}$ 及其形态 (除 F3 外) 存在显著相关关系 $(P<0.05)$, 而消落带上部仅有少量指标 与 $\mathrm{Cd}$ 及其形态存在相关性. 从消落带下部、消落带上部至背景点, 土壤 $\mathrm{Cd}$ 及其形态与理化性质的关系逐渐 减弱.

\section{3 讨论}

\section{1 高程与土地利用类型对消落带土壤 $\mathrm{Cd}$ 分布的影响}

自三峡水库蓄水以来,许多研究调查了三峡库区消落带土壤 $\mathrm{Cd}$ 随高程变化的分布特征 ${ }^{[28-30]}$, 对比这些 研究可以发现, 消落带土壤 $\mathrm{Cd}$ 的分布特征随着蓄水时间的增加发生明显变化. 在三峡水库蓄水初期, 张艳 敏等 ${ }^{[28]}$ 发现库区消落带上部 $(165 \sim 175 \mathrm{~m})$ 土壤 $\mathrm{Cd}$ 含量显著高于中部 (155 165 m) 和下部 (145 155 m), 并认为这一现象是由于消落带上部离城区更近, 受人类活动影响更大所导致的. 王业春等 ${ }^{[29]}$ 于 2010 年调查 了消落带 $160 \mathrm{~m}$ 和 $170 \mathrm{~m}$ 高程土壤 $\mathrm{Cd}$ 的分布特征, 发现虽然这两个高程淹水时间差异较大 (分别 334 天和 90 天), 但土壤 $\mathrm{Cd}$ 含量并无显著性差异, 认为这一现象是由于采样点淹水时间有限, 江水没能对消落带 $\mathrm{Cd}$ 的分布产生显著的影响. 杨丹等 ${ }^{[30]}$ 于 2014 年在忠县段消落带的研究显示, $164 \mathrm{~m}$ 高程处土壤 $\mathrm{Cd}$ 含量显著 高于 170 和 $176 \mathrm{~m}$ 高程, 并认为这是 $164 \mathrm{~m}$ 处淹水时间长、泥沙颗粒细且沉积速率快、对 $\mathrm{Cd}$ 的吸附能力更强 所导致的. 我们的研究同样发现, 消落带下部 $(150 \sim 160 \mathrm{~m}$ ) 土壤中 $\mathrm{Cd}$ 及其形态的含量显著高于消落带上部 $(165 \sim 175 \mathrm{~m})$. 根据不同淹水时间下消落带土壤 $\mathrm{Cd}$ 的研究结果可知, 随着三峡水库蓄水时间的增加, 土壤 $\mathrm{Cd}$ 在消落带累积的重点区域发生了明显改变, 即, 由消落带上部转变为下部, 这可能是长期以来库区水位变 化导致的泥沙沉降以及区域人类活动共同影响的结果. 
表 2 消落带土壤 $\mathrm{Cd}$ 及其各形态含量的分布特征

Tab.2 Characteristics of Cd content and its fractions in the riparian soils

\begin{tabular}{|c|c|c|c|c|c|c|}
\hline \multirow{2}{*}{ 土地利用类型 } & \multirow{2}{*}{ 高程/m } & \multicolumn{5}{|c|}{ 含量/( mg/kg) } \\
\hline & & $\mathrm{Cd}$ & Cd-F1 & $\mathrm{Cd}-\mathrm{F} 2$ & Cd-F3 & $\mathrm{Cd}-\mathrm{F} 4$ \\
\hline \multirow[t]{7}{*}{ 农田 } & 150 & $0.973 \pm 0.057^{\mathrm{a}}$ & $0.501 \pm 0.044^{\mathrm{a}}$ & $0.190 \pm 0.013^{\mathrm{a}}$ & $0.039 \pm 0.008^{\mathrm{ab}}$ & $0.241 \pm 0.010^{\mathrm{ab}}$ \\
\hline & 155 & $0.950 \pm 0.098^{\mathrm{a}}$ & $0.467 \pm 0.059^{\mathrm{a}}$ & $0.178 \pm 0.023^{\mathrm{a}}$ & $0.034 \pm 0.007^{\mathrm{ab}}$ & $0.270 \pm 0.042^{\mathrm{a}}$ \\
\hline & 160 & $0.901 \pm 0.089^{\mathrm{a}}$ & $0.436 \pm 0.050^{\mathrm{a}}$ & $0.188 \pm 0.019^{\mathrm{a}}$ & $0.057 \pm 0.009^{\mathrm{a}}$ & $0.218 \pm 0.030^{\mathrm{ab}}$ \\
\hline & 165 & $0.300 \pm 0.051^{\mathrm{b}}$ & $0.086 \pm 0.026^{\mathrm{b}}$ & $0.048 \pm 0.011^{\mathrm{b}}$ & $0.016 \pm 0.006^{\mathrm{b}}$ & $0.148 \pm 0.026^{\mathrm{bc}}$ \\
\hline & 170 & $0.179 \pm 0.008^{b}$ & $0.029 \pm 0.012^{\mathrm{b}}$ & $0.028 \pm 0.008^{b}$ & $0.015 \pm 0.006^{\mathrm{b}}$ & $0.105 \pm 0.019^{c}$ \\
\hline & 175 & $0.271 \pm 0.034^{\mathrm{b}}$ & $0.068 \pm 0.017^{\mathrm{b}}$ & $0.059 \pm 0.010^{\mathrm{b}}$ & $0.019 \pm 0.008^{b}$ & $0.124 \pm 0.017^{\mathrm{c}}$ \\
\hline & 180 & $0.273 \pm 0.049$ & $0.107 \pm 0.022$ & $0.058 \pm 0.008^{\mathrm{B}}$ & $0.011 \pm 0.004$ & $0.097 \pm 0.023$ \\
\hline \multirow{6}{*}{ 林地 } & 150 & $1.063 \pm 0.016^{\mathrm{a}}$ & $0.531 \pm 0.012^{\mathrm{a}}$ & $0.220 \pm 0.003^{\mathrm{a}}$ & $0.022 \pm 0.007$ & $0.289 \pm 0.010^{\mathrm{ab}}$ \\
\hline & 155 & $1.107 \pm 0.023^{\mathrm{a}}$ & $0.534 \pm 0.015^{\mathrm{a}}$ & $0.223 \pm 0.005^{\mathrm{a}}$ & $0.020 \pm 0.005$ & $0.328 \pm 0.015^{\mathrm{a}}$ \\
\hline & 160 & $0.480 \pm 0.080^{\mathrm{b}}$ & $0.201 \pm 0.038^{\mathrm{b}}$ & $0.061 \pm 0.021^{\mathrm{b}}$ & - & $0.215 \pm 0.021^{b c}$ \\
\hline & 165 & $0.526 \pm 0.037^{\mathrm{b}}$ & $0.196 \pm 0.012^{\mathrm{b}}$ & $0.067 \pm 0.014^{\mathrm{b}}$ & - & $0.260 \pm 0.031^{\mathrm{ab}}$ \\
\hline & 170 & $0.292 \pm 0.009^{c}$ & $0.105 \pm 0.001^{c}$ & $0.021 \pm 0.005^{\mathrm{b}}$ & - & $0.162 \pm 0.005^{c}$ \\
\hline & 180 & $0.335 \pm 0.028$ & $0.143 \pm 0.006$ & $0.049 \pm 0.010^{\mathrm{B}}$ & - & $0.141 \pm 0.012$ \\
\hline \multirow[t]{7}{*}{ 果园 } & 150 & $1.303 \pm 0.022^{\mathrm{a}}$ & $0.686 \pm 0.022^{\mathrm{a}}$ & $0.306 \pm 0.016^{\mathrm{a}}$ & $0.059 \pm 0.003$ & $0.250 \pm 0.018^{\mathrm{a}}$ \\
\hline & 155 & $1.064 \pm 0.086^{\mathrm{b}}$ & $0.531 \pm 0.055^{\mathrm{b}}$ & $0.222 \pm 0.024^{\mathrm{b}}$ & $0.031 \pm 0.012$ & $0.279 \pm 0.012^{\mathrm{a}}$ \\
\hline & 160 & $1.142 \pm 0.064^{\mathrm{b}}$ & $0.584 \pm 0.022^{\mathrm{b}}$ & $0.249 \pm 0.026^{\mathrm{b}}$ & $0.045 \pm 0.012$ & $0.262 \pm 0.013^{\mathrm{a}}$ \\
\hline & 165 & $0.271 \pm 0.010^{c}$ & $0.072 \pm 0.010^{\mathrm{c}}$ & $0.079 \pm 0.001^{c}$ & - & $0.117 \pm 0.011^{\mathrm{b}}$ \\
\hline & 170 & $0.215 \pm 0.011^{\mathrm{c}}$ & $0.082 \pm 0.004^{c}$ & $0.052 \pm 0.001^{\mathrm{c}}$ & - & $0.078 \pm 0.007^{b}$ \\
\hline & 175 & $0.233 \pm 0.006^{c}$ & $0.103 \pm 0.009^{c}$ & $0.039 \pm 0.003^{c}$ & - & $0.087 \pm 0.003^{b}$ \\
\hline & 180 & $0.297 \pm 0.043$ & $0.129 \pm 0.018$ & $0.060 \pm 0.009^{\mathrm{B}}$ & - & $0.104 \pm 0.018$ \\
\hline \multirow[t]{6}{*}{ 村庄 } & 150 & $0.953 \pm 0.052^{\mathrm{a}}$ & $0.474 \pm 0.040^{\mathrm{a}}$ & $0.186 \pm 0.009^{\mathrm{b}}$ & $0.105 \pm 0.001^{\mathrm{a}}$ & $0.187 \pm 0.006^{\mathrm{a}}$ \\
\hline & 155 & $1.062 \pm 0.074^{\mathrm{a}}$ & $0.588 \pm 0.022^{\mathrm{a}}$ & $0.236 \pm 0.020^{\mathrm{a}}$ & $0.099 \pm 0.006^{\mathrm{a}}$ & $0.137 \pm 0.064^{\mathrm{ab}}$ \\
\hline & 160 & $0.884 \pm 0.041^{\mathrm{a}}$ & $0.441 \pm 0.043^{\mathrm{a}}$ & $0.159 \pm 0.004^{b}$ & $0.081 \pm 0.006^{\mathrm{b}}$ & $0.202 \pm 0.009^{\mathrm{a}}$ \\
\hline & 165 & $0.283 \pm 0.085^{\mathrm{b}}$ & $0.152 \pm 0.055^{b}$ & $0.057 \pm 0.002^{\mathrm{c}}$ & $0.026 \pm 0.004^{\mathrm{c}}$ & $0.046 \pm 0.027^{b}$ \\
\hline & 170 & $0.142 \pm 0.009^{\mathrm{b}}$ & $0.061 \pm 0.008^{b}$ & $0.017 \pm 0.001^{\mathrm{d}}$ & $0.015 \pm 0.000^{\mathrm{c}}$ & $0.048 \pm 0.003^{b}$ \\
\hline & 180 & $0.625 \pm 0.213$ & $0.216 \pm 0.087$ & $0.157 \pm 0.042^{A}$ & $0.052 \pm 0.018$ & $0.199 \pm 0.067$ \\
\hline
\end{tabular}

小写字母代表同一土地利用类型下 $\mathrm{Cd}$ 及其形态的含量在高程间存在显著性差异, $P<0.05$; 大写字母代表背景点 $\mathrm{Cd}$ 及其 形态的含量在土地利用类型间存在显著性差异, $P<0.05$; 一代表元素未检出, 仪器检出限 $0.005 \mu \mathrm{g} / \mathrm{L}$.

水库消落带兼具陆地生态系统和水体生态系统的特点, 是土壤侵蚀与泥沙沉降发生的重要场所, 已有 研究表明, 三峡库区消落带下部的泥沙主要来自雨季河流泥沙的沉降 ${ }^{24]}$, 而消落带上部泥沙则来自旱季河 岸土壤侵蚀. 河流泥沙的长距离传输和沉积、当地河岸和土壤侵蚀的交互作用是形成当前消落带土壤中 $\mathrm{Cd}$ 及其形态海拔分布的主要因素. 本研究中, 村庄附近土壤中 $\mathrm{Cd}$ 含量 $(0.63 \pm 0.21 \mathrm{mg} / \mathrm{kg})$ 是其它土地利用类 型下的 2 3 倍 (图 2), 说明消落带上方的人类活动引起了当地土壤中 $\mathrm{Cd}$ 的累积, 但是并没有直接导致消落 带土壤中 $\mathrm{Cd}$ 的明显富集 (图 2, 表 2). 此外,在不同土地利用类型下消落带土壤中 $\mathrm{Cd}$ 及其可交换态及碳酸 盐结合态含量均无显著性差异, 但 $\mathrm{Cd}$ 及其形态均与高程存在极显著的负相关关系 (表 $3, P<0.01$ ). 而且, 与 消落带下部 (物源主要为远距离传输的河流泥沙) 相比, 消落带上部土壤中 $\mathrm{Cd}$ 含量及其形态与主要元素含 量相关性较弱, 这一现象更接近于背景点 (表 3). 这说明消落带上部土壤有相对于下部更复杂的来源, 可能 受到了坡岸土壤侵蚀的影响, 这与已有研究观点一致 ${ }^{[24,31]}$. 消落带上方土地利用方式对消落带土壤 $\mathrm{Cd}$ 的贡 献不大的原因有两个. 首先, 消落带上方的人类活动并未直接向消落带输人污染物, 当地居民所排放的生活 污水和农业面源排放等主要通过大的沟渠排放至干流水体中, 而未直接到达消落带土壤中. 其次, 虽然消落 带上方的土壤在降雨冲刷作用下会进人消落带, 但是在经历了侵蚀一搬运一沉降一混合这一过程后, 不同土 地利用类型下土壤 $\mathrm{Cd}$ 含量的差异可能被弱化. 
表 3 土壤理化性质及高程与 $\mathrm{Cd}$ 及其各形态含量间的相关关系

Tab.3 Spearman correlation of Cd content and its fractions with soil physicochemical properties and altitude

\begin{tabular}{|c|c|c|c|c|c|c|c|c|c|c|c|c|c|c|}
\hline \multirow{2}{*}{ 采样点 } & \multirow{2}{*}{1} & \multirow{2}{*}{ 高程 } & \multirow{2}{*}{$\mathrm{pH}$} & \multirow{2}{*}{ LOI } & \multicolumn{3}{|c|}{ 粒度组成 } & \multicolumn{7}{|c|}{ 元素浓度 } \\
\hline & & & & & 黍粒 & 粉粒 & 砂粒 & $\mathrm{Al}$ & $\mathrm{Ca}$ & $\mathrm{Fe}$ & $\mathrm{Mg}$ & Mn & $\mathrm{P}$ & $\mathrm{Ti}$ \\
\hline 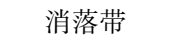 & $\mathrm{Cd}$ & $-0.82^{* *}$ & -0.04 & $-0.25 *$ & $* 0.56^{* *}$ & * $0.72^{* * *}$ & -0.81 ** & ** 0.73 ** & $* 0.77$ *** & * 0.81 ** & * 0.84 *** & 0.84 ** & * 0.91 ** & $0.78^{* *}$ \\
\hline$(150 \sim 175 \mathrm{~m})$ & Cd-F1 & $-0.83^{* *}$ & * -0.06 & $-0.27 *$ & * $0.57^{\text {*** }}$ & * $0.68^{* *}$ & $-0.79^{* *}$ & ** 0.63 ** & * 0.74 *** & * $0.73^{* *}$ & * 0.79 *** & 0.79 ** & $* 0.88^{* * *}$ & $0.74^{* * *}$ \\
\hline \multirow[t]{3}{*}{$N=66$} & Cd-F2 & $-0.80^{* * *}$ & -0.02 & -0.17 & $0.66^{* *}$ & * $0.65^{* *}$ & -0.78 ** & $* * 0.74 * *$ & * $0.77^{* * *}$ & $0.79 * *$ & * $0.83^{\text {*** }}$ & 0.83 ** & $* 0.86^{* * *}$ & $0.72^{\text {*** }}$ \\
\hline & Cd-F3 & $-0.57^{* *}$ & -0.07 & $-0.28 *$ & ** 0.64 *** & $0.26^{*}$ & $-0.48 * *$ & ** $0.43^{* *}$ & $* 0.64$ *** & * $0.49^{* * *}$ & * 0.55 ** & $0.62 * *$ & $* 0.49 * *$ & $0.56^{* *}$ \\
\hline & Cd-F4 & $-0.68^{* *}$ & * 0.01 & -0.17 & $0.33^{* *}$ & * 0.74 ** & $-0.71 * *$ & $* * 0.70 * *$ & $* 0.62 * *$ & * $0.75^{* * *}$ & * 0.73 *** & $0.69 * *$ & $* 0.76^{* *}$ & $0.69^{* *}$ \\
\hline 消落带下部 & $\mathrm{Cd}$ & - & -0.06 & -0.04 & 0.14 & $0.49^{\text {*** }}$ & -0.57 ** & ** 0.42 ** & * $0.36^{*}$ & $0.69^{* *}$ & * $0.53^{\text {*** }}$ & $0.78^{* *}$ & * $0.89^{* *}$ & 0.23 \\
\hline$(150 \sim 160 \mathrm{~m})$ & Cd-F1 & - & -0.13 & -0.08 & 0.22 & $0.44^{* * *}$ & -0.57 ** & ** 0.30 * & $0.41^{* *}$ & $0.57^{* * *}$ & * $0.48^{\text {*** }}$ & $0.71 * *$ & $* 0.80^{* *}$ & 0.24 \\
\hline \multirow[t]{3}{*}{$N=36$} & Cd-F2 & - & 0.04 & 0.04 & 0.21 & $0.40^{* * *}$ & -0.48 ** & $* * 0.49$ ** & $* 0.22$ & $0.63^{\text {** }}$ & * 0.38 * & $0.69^{* *}$ & $* 0.78$ ** & 0.10 \\
\hline & Cd-F3 & - & -0.12 & -0.18 & 0.23 & -0.26 & 0.13 & -0.21 & 0.25 & -0.17 & -0.02 & 0.18 & 0.18 & 0.21 \\
\hline & $\mathrm{Cd}-\mathrm{F} 4$ & - & 0.08 & 0.17 & -0.10 & $0.61^{\text {** }}$ & -0.60 ** & ** 0.52 ** & 0.17 & $0.67^{* *}$ & * $0.48^{\text {*** }}$ & $0.41^{* * *}$ & * $0.54^{* * *}$ & 0.14 \\
\hline \multirow{5}{*}{$\begin{array}{c}\text { 消落带上部 } \\
(165 \sim 175 \mathrm{~m}) \\
N=30\end{array}$} & $\mathrm{Cd}$ & - & 0.06 & -0.02 & -0.25 & 0.28 & -0.16 & 0.17 & 0.13 & 0.15 & 0.29 & 0.06 & $0.61^{\text {*** }}$ & 0.25 \\
\hline & Cd-F1 & - & 0.05 & -0.07 & -0.30 & 0.09 & 0.01 & -0.28 & -0.23 & -0.26 & -0.08 & $-0.33^{*}$ & $0.45^{\text {** }}$ & -0.06 \\
\hline & Cd-F2 & - & 0.01 & 0.10 & 0.28 & 0.05 & -0.16 & 0.23 & $0.32 *$ & 0.12 & $0.45^{\text {** }}$ & 0.08 & 0.29 & 0.00 \\
\hline & Cd-F3 & - & 0.04 & -0.12 & $0.45^{* *}$ & $* 0.51^{* *}$ & 0.16 & -0.06 & 0.18 & -0.11 & -0.17 & -0.06 & $-0.64^{* *}$ & -0.29 \\
\hline & Cd-F4 & - & -0.04 & 0.05 & $-0.43^{\text {*** }}$ & $0.57^{* *}$ & -0.27 & $0.39^{*}$ & 0.19 & $0.41^{\text {** }}$ & 0.34 * & $0.37^{*}$ & $0.65^{\text {** }}$ & $0.60^{\text {** }}$ \\
\hline \multirow{5}{*}{$\begin{array}{c}\text { 背景点 } \\
(180 \mathrm{~m}) \\
N=12\end{array}$} & $\mathrm{Cd}$ & - & -0.04 & 0.03 & -0.28 & 0.11 & -0.01 & 0.39 & 0.14 & 0.50 & 0.21 & 0.23 & 0.38 & $0.60^{*}$ \\
\hline & $\mathrm{Cd}-\mathrm{F} 1$ & - & 0.14 & -0.05 & -0.31 & 0.25 & -0.06 & 0.49 & 0.36 & $0.66^{* *}$ & $* 0.40$ & 0.19 & $0.72^{\text {** }}$ & 0.72 ** \\
\hline & Cd-F2 & - & -0.14 & 0.06 & -0.05 & 0.08 & -0.06 & 0.51 & 0.25 & $0.57^{*}$ & 0.36 & 0.21 & 0.48 & $0.56^{*}$ \\
\hline & Cd-F3 & - & -0.01 & 0.37 & 0.29 & -0.46 & 0.25 & 0.14 & 0.18 & -0.11 & 0.05 & 0.28 & -0.16 & -0.28 \\
\hline & Cd-F4 & - & -0.04 & -0.15 & -0.33 & 0.14 & -0.01 & 0.21 & 0.09 & 0.36 & 0.09 & 0.15 & 0.32 & 0.50 \\
\hline
\end{tabular}

* 代表存在显著性差异, $P<0.05 ; * *$ 代表存在极显著性差异, $P<0.01$.

消落带土壤中 $\mathrm{Cd}$ 及其形态的海拔分布主要是通过水位变化导致的泥沙输人造成的. 消落带被含沙水 流淹没的过程中,泥沙及其所携带的 $\mathrm{Cd}$ 一同在消落带累积. 已有大量研究表明 ${ }^{[24,31-32]}$, 由于旱季(夏季)消 落带水位较低, 此时库区强降雨过程频发, 江水含细颗粒悬移质较多, 加之消落带下部被含沙水流淹没时间 较长, 从而消落带下部是泥沙淤积的主要场所. 由于库区水体的扰动作用, 消落带土壤总是受到沉降与再悬 浮两个过程的影响, 消落带泥沙年沉积速率变化范围为 $0.5 \sim 10 \mathrm{~cm} / \mathrm{a}$, 且沉积速率随高程增加而显著降低, 泥沙净沉积主要发生在 $145 \sim 168 \mathrm{~m}$ 高程 ${ }^{[24]}$. 因此, 泥沙与 $\mathrm{Cd}$ 在消落带的分布同步变化, 表现为随着高程增 加, 泥沙沉积速率下降 ${ }^{[24]}$ 且细颗粒物质占比降低 (表 1), Cd 及其各形态的含量同步下降. 综上, 目前控制三 峡库区消落带土壤中 $\mathrm{Cd}$ 及其形态分布的主要因素与水库的水位调控模式密切相关, 而消落带及其上方的 土地利用方式对其影响较弱.

\section{2 理化性质对 Cd 分布的影响}

自三峡水库运行以来, 消落带经历了长期的水位涨落, 消落带理化性质较蓄水前发生了巨大变化 ${ }^{[33]}$, 例 如, 土壤 $\mathrm{pH}$ 趋于稳定, 呈中性, 有机质含量降低等 (表 1). 土壤理化性质的变化直接影响到土壤 $\mathrm{Cd}$ 形态的 转变以及之后的累积或释放. 土壤粒度组成是影响库区消落带 $\mathrm{Cd}$ 分布的主要因素之一. 本研究中, 消落带 土壤 $\mathrm{Cd}$ 与黏粒、粉粒呈显著正相关而与砂粒呈显著负相关 (表 3), 这与其它研究结果一致 ${ }^{[19,34]}$. 不同粒级 颗粒的矿物组成、比表面积和自由能存在差异,进而导致其与重金属离子的结合能力不同 ${ }^{[35-36]}$. 通常认为粒 径 $\leqslant 64 \mu \mathrm{m}$ 的颗粒, 即黏粒与粉粒, 其比表面积大且具有较高的黏土矿物、有机质和铁锰氧化物含量, 能够吸 附固定更多的重金属 ${ }^{[37]}$. 此外, 土壤 $\mathrm{Cd}$ 的形态分布也受到粒度组成的影响. 一方面, 由于受到总 $\mathrm{Cd}$ 含量的 影响, 土壤中细颗粒物质占比越大, 各形态 $\mathrm{Cd}$ 含量均随总 $\mathrm{Cd}$ 含量的升高而增大; 另一方面, 由于残渣态 $\mathrm{Cd}$ 与非残渣态 $\mathrm{Cd}$ 在土壤中的赋存形式不同,粒度组成更粗的土壤其残渣态 $\mathrm{Cd}$ 的占比越大 ${ }^{[38]}$.

消落带土壤主要元素与 Cd 及其形态间具有显著的正相关关系 (表 3),一方面,这与它们物源的相似性 
有关. 泥沙在消落带沉积的过程中不仅向消落带输人 $\mathrm{Cd}$, 同样也造成其它元素的大量沉降. 对于没有泥沙 输人的消落带上方样点 ( $180 \mathrm{~m}$ 高程), 除了与 $\mathrm{P} 、 \mathrm{Ti}$ 和 $\mathrm{Fe}$ 外, $\mathrm{Cd}$ 与其它主要元素含量关系不显著, 这说明 $\mathrm{Cd}$ 的来源具有复杂性, 可能包括母质风化、面源输人、大气沉降等. 例如, 土壤 $\mathrm{Cd}$ 与土壤母质参比元素 $\mathrm{Ti}$ 存在 显著性相关关系 $\left(R^{2}=0.54, P<0.01\right)$, 说明母质风化是土壤 $\mathrm{Cd}$ 的来源之一; 土壤 $\mathrm{P}$ 与可交换态及碳酸盐结 合态 $\mathrm{Cd}$ 存在极显著的正相关关系 $\left(R^{2}=0.72, P<0.01\right)$, 这可能说明农业化肥的使用可是库区土壤 $\mathrm{Cd}$ 的来 源之一 ${ }^{[39]}$. 另一方面, 消落带下部土壤 $\mathrm{Cd}$ 与主要元素 (尤其是 $\mathrm{Al} 、 \mathrm{Ca} 、 \mathrm{Fe} 、 \mathrm{Mg} 、 \mathrm{Mn}$ ) 间关系密切, 说明土壤碳 酸盐矿物和金属氧化物/氢氧化物对 $\mathrm{Cd}$ 具有调控作用,而土壤 $\mathrm{Cd}$ 的形态与这些元素间的关系同样支持了 这一结论 $(\mathrm{Al} 、 \mathrm{Fe}$ 和 $\mathrm{Mn}$ 与 $\mathrm{Cd}-\mathrm{F} 2$ 含量呈极显著正相关,表 3).

研究发现,消落带土壤 $\mathrm{pH}$ 与 $\mathrm{Cd}$ 含量无显著相关关系, 这可能是由于库区消落带受到反复的淹水与暴 露过程. 打破了正常的水土化学过程, 使土壤 $\mathrm{pH}$ 整体偏中性, 减弱了 $\mathrm{pH}$ 与 $\mathrm{Cd}$ 之间的联系, 而在库区人类 活动影响下, 土壤 $\mathrm{Cd}$ 随着水库运行时间的增加其累积程度不断增加, 从而造成两者之间没有呈现出显著的 相关关系, 这与程瑞梅等 ${ }^{[33]}$ 的研究结果一致. 此外, 消落带土壤有机质与 $\mathrm{Cd}$ 呈显著负相关, 这可能是由于 消落带下部长期淹水, 缺少植被对有机质的有效补给, 而消落带上方在退水阶段通常发育有草本植物 (如狗 牙根等), 因而消落带上方土壤有机质含量高于消落带下方, 呈现出与 $\mathrm{Cd}$ 相反的趋势. 消落带土壤有机物及 硫化物结合态 $\mathrm{Cd}$ 所占比例 $(5.13 \% \pm 0.53 \%)$ 较低, 这可能是由于消落带有机质含量较低所造成的.

\section{4 结论}

三峡库区丰都一忠县段消落带土壤 $\mathrm{Cd}$ 含量为 $0.65 \pm 0.04 \mathrm{mg} / \mathrm{kg}$, 并以可交换态及碳酸盐结合态 $(41.21 \%)$ 和残渣态 $(34.71 \%)$ 为主. 空间上, 土壤 Cd 及其形态均随消落带高程上升而显著降低 $(P<0.05)$, 但是在不同土地利用类型间整体上不存在显著性差异. 库区水位变化导致的泥沙沉积是控制消落带土壤 $\mathrm{Cd}$ 空间分布的主要因素,而消落带上方人类活动对其直接的贡献较弱. 土壤粒度组成是土壤 $\mathrm{Cd}$ 及其各形态含 量分布最主要的影响因素, 而土壤 $\mathrm{pH}$ 和有机质对 $\mathrm{Cd}$ 的分布影响较弱. 将来需要重点关注三峡水库反季节 水位调控下沉积环境的变化特征, 以及土壤 $\mathrm{Cd}$ 动态迁移和形态转化对水环境的影响.

\section{5 参考文献}

[ 1 ] Xu ZC, Yang XY, Chen GH et al. Evaluation of the heavy metals contamination and its potential ecological risk of the sediments on Beijiang River's upper and middle reaches. Environmental Science, 2009, 30(11): 3262-32688. [许振成, 杨 晓云, 陈桂华等. 北江中上游底泥重金属污染及其潜在生态危害评价. 环境科学, 2009, 30(11): 3262-3268.]

[ 2 ] Dong JQ, Zhang HZ, Wang JN et al. Modeling of cadmium speciation in Longjiang River during an emergent environmental incident. China Environmental Science, 2015, 35(10): 3046-3052. [董璟琦, 张红振, 王金南等. 龙江河突发环境事 件河流镉污染化学形态模拟. 中国环境科学, 2015, 35(10): 3046-3052.]

[ 3 ] Wang MY, Zhang L, Qin YW et al. Speciation of heavy metals in sediments from Xiang River and analysis of their environmental factors. Acta Scientiae Circumstantiae, 2011, 31(11): 2447-2458. [王鸣宇, 张雷, 秦延文等. 湘江表层沉积物 重金属的赋存形态及其环境影响因子分析. 环境科学学报, 2011, 31(11)：2447-2458.]

[ 4 ] Olivares-Rieumont S, de la Rosa D, Lima L et al. Assessment of heavy metal levels in Almendares River sediments-Havana City, Cuba. Water Research, 2005, 39: 3945-3953.

[ 5 ] Zhao HT, Li XY, Wang XM et al. Grain size distribution of road-deposited sediment and its contribution to heavy metal pollution in urban runoff in Beijing, China. Journal of Hazardous Materials, 2010, 183(1) : 203-210.

[ 6 ] Shao ST, Deng HM, Song YX et al. Adsorption of thallium and cadmium by wetland sediments. Environmental Chemistry, 2018, 37(7) : 1534-1544. [邵思婷, 邓红梅, 宋永欣等. 湿地沉积物对铊、镉的吸附性能. 环境化学, 2018, 37 (7) : 1534-1544.]

[ 7 ] Du LG, Rinklebe J, Vandecasteele B et al. Trace metal behaviour in estuarine and riverine floodplain soils and sediments : A review. Science of the Total Environment, 2009, 407(13): 3972-3985.

[ 8 ] Klerks PL, Felder DL, Strasser K et al. Effects of ghost shrimp on zinc and cadmium in sediments from Tampa Bay. Marine Chemistry, 2007, 104(1): 17-26.

[ 9 ] Peng JF, Song YH, Yuan P et al. The remediation of heavy metals contaminated sediment. Journal of Hazardous Materi- 
als, 2009, 161(2) : 633-640.

[10] Zhang C, Yu ZG, Zeng GM et al. Effects of sediment geochemical properties on heavy metal bioavailability. Environment International, 2014, 73: 270-281.

[11] Di Toro DM, Mahony JD, Hansen DJ et al. Acid volatile sulfide predicts the acute toxicity of cadmium and nickel in sediments. Environmental Science \& Technology, 1992, 26(1) : 96-101. DOI: 10.1021/es00025a009.

[12] Di Toro DM, Mahony JD, Hansen DJ et al. Toxicity of cadmium in sediments: The role of acid volatile sulfide. Environmental Toxicology and Chemistry, 1990, 9(12): 1487-1502. DOI: 10.1002/etc.5620091208.

[13] Gregory SV, Swanson FJ, Mckee WA et al. An ecosystem perspective of riparian zones. BioScience, 1991, 41 (8): 540-551.

[14] Bao YH, Gao P, He XB. The water-level fluctuation zone of Three Gorges Reservoir - A unique geomorphological unit. Earth-Science Reviews, 2015, 150: 14-24.

[15] Friedl G, Wüsat A. Disrupting biogeochemical cycles—Consequences of damming. Aquatic Sciences, 2002, 64(1) : 55-65.

[16] Ye C, Li SY, Zhang YL et al. Assessing soil heavy metal pollution in the water-level-fluctuation zone of the Three Gorges Reservoir, China. Journal of Hazardous Materials, 2011, 191(1-3) : 366-372.

[17] Wei X, Han LF, Gao B et al. Distribution, bioavailability, and potential risk assessment of the metals in tributary sediments of Three Gorges Reservoir: The impact of water impoundment. Ecological Indicators, 2016, 61 : 667-675.

[18] Zhu H, Bing HJ, Wu YH et al. The spatial and vertical distribution of heavy metal contamination in sediments of the Three Gorges Reservoir determined by anti-seasonal flow regulation. Science of the Total Environment, 2019, 664: 79-88.

[19] Bing HJ, Zhou J, Wu YH et al. Current state, sources, and potential risk of heavy metals in sediments of Three Gorges Reservoir, China. Environmental Pollution, 2016, 214: 485-496. DOI: 10.1016/j.envpol.2016.04.062

[20] Bing HJ, Wu YH, Zhou J et al. Spatial variation of heavy metal contamination in the riparian sediments after two-year flow regulation in the Three Gorges Reservoir, China. Science of the Total Environment, 2019, 649: 1004-1016.

[21] Wang XX, Bing HJ, Wu YH et al. Distribution and potential eco-risk of chromium and nickel in sediments after impoundment of Three Gorges Reservoir, China. Human and Ecological Risk Assessment: An International Journal, 2016, 23(1) : 172-185.

[22] Wei X, Han LF, Gao B et al. Distribution, bioavailability, and potential risk assessment of the metals in tributary sediments of Three Gorges Reservoir: The impact of water impoundment. Ecological Indicators, 2016, 61: 667-675.

[23] Xu DY, Gao B, Gao L et al. Characteristics of cadmium remobilization in tributary sediments in Three Gorges Reservoir using chemical sequential extraction and DGT technology. Environmental Pollution, 2016, 218: 1094-1101.

[24] Tang Q, Bao YH, He XB et al. Sedimentation and associated trace metal enrichment in the riparian zone of the Three Gorges Reservoir, China. Science of the Total Environment, 2014, 479-480: 258-266.

[25] Liu EF, Zhang EL, Li K et al. Historical reconstruction of atmospheric lead pollution in central Yunnan province, southwest China: an analysis based on lacustrine sedimentary records. Environmental Science and Pollution Research, 2013,20 (12) : 8739-8750.

[26] Rauret G, López-Sánchez JF, Sahuquillo A et al. Improvement of the BCR three step sequential extraction procedure prior to the certification of new sediment and soil reference materials. Journal of Environmental Monitoring, 1999, 1(1): 57-61. DOI: $10.1039 / \mathrm{A} 807854 \mathrm{H}$.

[27] Pueyo M, Mateu J, Rigol A et al. Use of the modified BCR three-step sequential extraction procedure for the study of trace element dynamics in contaminated soils. Environmental Pollution, 2008, 152(2) : 330-341.

[28] Zhang YM, Liu H, Wei SQ et al. Investigation and assessment of heavy metal pollution from various vertical heights in water flooding fluctuation zone of Three Gorges Reservoir Areas. Chinese Agricultural Science Bulletin, 2011, 27( 8) : 317 322. [张艳敏, 刘海, 魏世强等. 三峡库区消落带不同垂直高程土壤重金属污染调查与评价. 中国农学通报, $2011,27(8): 317-322$.

[29] Wang YC, Lei B, Yang SM et al. Concentrations and pollution assessment of soil heavy metals at different water-level altitudes in the draw-down areas of the Three Gorges Reservoir. Environmental Science, 2012,(2): 612-617. [王业春, 雷 波, 杨三明等. 三峡库区消落带不同水位高程土壤重金属含量及污染评价. 环境科学, 2012,(2) : 612-617.]

[30] Yang D, Xie ZQ, Fan DY et al. The effect of water fluctuation on the contents of soil Cu, Zn, Cr and Cd at the riparian area of Three Gorges Reservoir. Journal of Natural Resources, 2018, 33(7) : 1283-1290. DOI:0.31497/zrzyxb.20170637. 
[杨丹, 谢宗强, 㚞大勇等. 三峡水库蓄水对消落带土壤 Cu、Zn、Cr、Cd 含量的影响. 自然资源学报, 2018, 33(7)： 1283-1290]

[31] Bao YH, Tang Q, He XB et al. Soil erosion in the riparian zone of the Three Gorges Reservoir, China. Hydrology Research, 2015, 46(2) : 198-201.

[32] Wang BY, Wen AB, Yan DC et al. Factors influencing sedimentation in the riparian zone of the Three Gorges Reservoir, China. Science of Soil and Water Conservation, 2016, 14(1): 12-20. [王彬俨, 文安邦, 严冬春等. 三峡水库干流消落 带泥沙沉积影响因素. 中国水土保持科学, 2016, 14(1): 12-20.]

[33] Cheng RM, Liu ZB, Xiao WF et al. Changes of soil chemical properties in typical hydro-fluctuation belt of Three Gorges Reservoir. Scientia Silvae Sinicae, 2017, 53(2) : 19-25. DOI:10.11707/j.1001-7488.20170203. [程瑞梅, 刘泽彬, 肖 文发等. 三峡库区典型消落带土壤化学性质变化. 林业科学, 2017, 53(2) : 19-25.]

[34] Ye C, Butler OM, Du M et al. Spatio-temporal dynamics, drivers and potential sources of heavy metal pollution in riparian soils along a 600 kilometre stream gradient in Central China. Science of the Total Environment, 2019, 651: 1935-1945.

[35] Liu EF, Shen J, Zhu YX. The influence of grain size composition on metal content in the sediment: the case of core sediment in Lake Taihu. J Lake Sci, 2006, 18(4) : 363-368. DOI:10.18307/2006.0407. [刘恩峰, 沈吉, 朱育新. 沉积物 金属元素变化的粒度效应——太湖沉积岩芯为例. 湖泊科学, 2006, 18(4):363-368.]

[36] Wang FY, Chen JS. Relation of sediment characteristics to trace metal concentrations: A statistical study. Water Research, 2000, 34(2) : 694-698.

[37] Campana O, Blasco J, Simpson SL. Demonstrating the appropriateness of developing sediment quality guidelines based on sediment geochemical properties. Environmental Science \& Technology, 2013, 47 ( 13 ): 7483-7489. DOI: 10. 1021/es4009272.

[38] Murray KS, Cauvet D, Lybeer M et al. Particle size and chemical control of heavy metals in bed sediment from the rouge river, Southeast Michigan. Environmental Science \& Technology, 1999, 33(7) : 987-992. DOI: 10.1021/es9807946.

[39] Huang QQ, Yu Y, Wan YN et al. Effects of continuous fertilization on bioavailability and fractionation of cadmium in soil and its uptake by rice( Oryza sativa L.). Journal of Environmental Management, 2018, 215: 13-21. 